\title{
Assessing Eulerian-Lagrangian simulations of dense solid-liquid suspensions settling under gravity
}

\author{
J.J. Derksen \\ School of Engineering, University of Aberdeen, Aberdeen, UK \\ jderksen@abdn.ac.uk
}

Submitted to Computers \& Fluids (Special Issue TI2015), April 2016

Accepted: December 2016

\section{Abstract}

We study dense solid-liquid suspensions through numerical simulations. The liquid flow is solved by the lattice-Boltzmann method on a fixed (Eulerian), cubic, uniform grid. Spherical solid particles are tracked through that grid. Our main interest is in cases where the grid spacing and the particle diameter have the same order of magnitude $(d / \Delta=O(1))$. Critical issues then are the mapping operations that relate properties on the grid and properties of the particles, e.g. the local solids volume fraction seen by a particle, or the distribution of solid-to-liquid hydrodynamic forces over grid points adjacent to a particle. For assessing the mapping operations we compare results for particles settling under gravity in fully periodic, three-dimensional domains of simulations with $d / \Delta=O(1)$ to much higher resolved simulations $(d / \Delta=O(10))$ that do not require mapping. Comparisons are made in terms of average slip velocities as well as in terms of liquid and fluid velocity fluctuation levels. Solids volume fractions are in the range 0.3 to 0.5 , Reynolds numbers are of order 0.1 to 10 .

\section{Keywords}

Solid-liquid suspension, lattice-Boltzmann method, discrete particle method, hindered settling, two-way coupling, agitated suspensions 


\section{Introduction}

Engineering applications where solid particles are suspended in a liquid phase are abundant. An important class of applications - and the motivation for the research presented in this article - relates to industrial and pharmaceutical crystallization [1]. Other applications are sediment transport (e.g. in environmental flows [2]), catalytic slurry reactors [3], and hydrotransport in the mining industries [4]. Crystallization processes are often carried out in agitated tanks operated under turbulent conditions with high solids loading ( $20 \%$ solids by overall volume is not exceptional). The distribution of solids in the tank and therefore the hydrodynamic conditions individual crystals get exposed to are thought to be of influence on product quality: crystal size distribution, crystal shape (think of particle attrition due to frequent collisions), and crystal purity.

Computational methods are one way of enhancing our understanding of solids suspension processes in mixing tanks. Advances in numerical methodologies and - first and foremost - wide availability of high-performance computational resources make that more and more details and complexity can be accounted for in simulations. Specifically in the field of crystallization there are good reasons to invest computational effort in resolving the behavior of individual particles because the particles are the actual product and knowing their history in the course of their formation process (and the extent to which this history varies from crystal to crystal) is relevant. Among more, this notion has motivated research on Eulerian-Lagrangian descriptions of solid-liquid suspensions: Fluid flow is resolved in an Eulerian manner (i.e. on a fixed computational grid) and through this Eulerian flow field solid particles are tracked [5]. The specific situations we are interested in have particle sizes $(d)$ that are of the same order of magnitude as the Eulerian grid spacing $\Delta$, i.e. $d=O(\Delta)$. This is somewhere in between particle-resolved simulations $(d \gg \Delta)[6]$, and the cases more common in Eulerian-Lagrangian simulations that have $d \ll \Delta[7]$.

The situation we have in mind is that of a lab scale (order one liter) tank containing liquid and 300 $\mu \mathrm{m}$ size solid particles. If the solids volume fraction is some 10 to $20 \%$, the tank contains of the order of 
$10^{7}$ particles. Dealing with such numbers of particles in a simulation is very well feasible [8], resolving the liquid flow around each individual particle is, however, not that feasible. By not resolving the flow around each particle we do not want to use a simulation method that requires $d \ll \Delta$. This would make the Eulerian grid too coarse to e.g. perform a well-resolved large-eddy simulation of the liquid flow.

The aim of this paper is to describe and calibrate a numerical procedure for Eulerian-Lagrangian simulations of solid-liquid suspensions with particles and grid spacing having comparable size: $d=O(\Delta)$. As will become clear, the operation of exchanging information between particles (Lagrangian information) and the liquid flow on the grid (Eulerian information), this operation is usually referred to as mapping, requires numerical settings for which no closed theory is available. At the same time, these settings have a pronounced impact on the outcome of a simulation. In order to make objective choices for the numerical settings, simulations of sedimenting particles in fully periodic domains have been performed with the here proposed, unresolved particles procedure. The results are compared to results of particle-resolved simulations (already published, see [9]) of the same, fully periodic systems. Next to average relative velocities of particles with respect to liquid (i.e. superficial slip velocities), an important demand of the particle-unresolved simulations is that they are able to reproduce the velocity fluctuation levels (of particles and liquid) as observed in the benchmark particle-resolved simulations. This is not only important from a fundamental point of view, it also is from a (industrial crystallization) practical point of view: Fluctuations determine collisions between particles, and are highly influential to mass transfer between liquid and solid as e.g. occurs in reactive crystallization, or when crystals grow or dissolve in the liquid phase.

This paper is organized in the following order: First the fully periodic sedimentation configuration is discussed. We then give a brief account of the fully resolved simulation method that provides the benchmark results for assessing the much less $(d=O(\Delta))$ resolved ("particle-unresolved") simulations. The methodology for particle-unresolved simulations is discussed and subsequently assessed in detail. 
Before summarizing the results in the Conclusions section, we give an outlook on applying our particleunresolved numerical approach to agitation of a dense solid liquid suspension in a mixing tank.

\section{Flow system and simulation method}

\subsection{Flow system}

The system is a three-dimensional periodic domain of size $n x \times n y \times n z$ with $n x=2 n y=2 n z$. It contains a Newtonian liquid with density $\rho$ and kinematic viscosity $v$, and solid particles. The solid particles are monosized spheres with diameter $d$ and density $\rho_{s}$ larger than $\rho$. If there are $N$ spheres, the solids volume fraction is $\phi=N \frac{\pi d^{3}}{6 n x \cdot n y \cdot n z}$. This allows for the definition of the mixture density $\rho_{m} \equiv \phi \rho_{s}+(1-\phi) \rho$. A gravitational acceleration $g$ acts in the negative $x$-direction: $\mathbf{g}=-g \mathbf{e}_{\mathbf{x}}$. The net gravity force (gravity minus buoyancy) on each sphere has a magnitude $g\left(\rho_{s}-\rho_{m}\right) \pi d^{3} / 6=g(1-\phi)\left(\rho_{s}-\rho\right) \pi d^{3} / 6$ and acts in negative $x$-direction. To have a zero overall force on the periodic domain - and thus prevent the domain as a whole from accelerating - a body force (force per unit volume) in positive $x$-direction of $\mathbf{f}=g\left(\rho_{m}-\rho\right) \mathbf{e}_{\mathbf{x}}$ is applied to the liquid [10].

One way to characterize the system above is through the following set of dimensionless numbers: solids volume fraction $\phi$, density ratio $\rho_{s} / \rho$, aspect ratio $d / n x$, and the single-particle settling Reynolds number $\operatorname{Re}_{\infty} \equiv u_{\infty} d / v$ with $u_{\infty}$ the settling velocity that we determine from a force balance over a single particle in an infinite domain $g\left(\rho_{s}-\rho\right) \pi d^{3} / 6=\frac{1}{2} C_{D} \rho u_{\infty}^{2} \pi d^{2} / 4$. For the drag coefficient $C_{D}$ the Schiller-Naumann correlation [11] $C_{D}=24\left(1+0.15 \mathrm{Re}^{0.687}\right) / \mathrm{Re}$ is applied.

\subsection{Resolved simulations}

In previous papers $[9,10,12]$ we have studied systems like this by means of particle-resolved simulations. In such simulations the grid on which the liquid flow is solved is much finer than the size of the particles 
(typically the diameter $d$ spans 12 to 24 lattice spacings). This allows to explicitly impose the no-slip boundary condition on each spherical solid-liquid interface. The forces and torques on each particle that result from this procedure are used to update particle locations and velocities (translational as well as rotational). These moving no-slip conditions for the liquid provide an intimate coupling between liquid and solids dynamics. Details of the particle-resolved simulations, their methodology and performance, can be found in the papers referred to above.

\subsection{Unresolved simulations}

The results of particle-resolved simulations will be confronted with those of particle-unresolved simulations. Such unresolved simulations are the focus of this paper. As explained in the introduction, we will be dealing with dense suspensions $(\phi>0.2)$ in an Eulerian-Lagrangian manner. The Eulerian grid is

uniform and cubic with grid spacing $\Delta$. The spherical particles that move through this grid have a diameter comparable to $\Delta$; the range of diameters investigated is $0.75 \leq d / \Delta \leq 2$.

On the Eulerian grid the continuity equation and momentum balance for the liquid phase [10] are solved:

$$
\begin{gathered}
\frac{\partial}{\partial t}\left(\rho \phi^{c}\right)+\nabla \cdot\left(\rho \phi^{c} \mathbf{u}\right)=0 \\
\frac{\partial}{\partial t}\left(\rho \phi^{c} \mathbf{u}\right)+\nabla \cdot\left(\rho \phi^{c} \mathbf{u u}\right)=\phi^{c} \nabla \cdot \boldsymbol{\pi}+\mathbf{f}+\mathbf{f}_{\mathrm{s}}
\end{gathered}
$$

with $\phi^{c} \equiv 1-\phi$ the continuous phase (liquid) volume fraction, $\mathbf{u}$ the interstitial liquid velocity, $\boldsymbol{\pi}$ the liquid's stress tensor, and $\mathbf{f}_{\mathrm{s}}$ the force per unit volume the solid particles exert on the liquid. Equations 1 and 2 are rewritten in the following form

$$
\begin{gathered}
\frac{\partial \rho}{\partial t}+\nabla \cdot(\rho \mathbf{u})=-\frac{\rho}{\phi^{c}}\left[\frac{\partial \phi^{c}}{\partial t}+\mathbf{u} \cdot \nabla \phi^{c}\right] \\
\frac{\partial}{\partial t}(\rho \mathbf{u})+\nabla \cdot(\rho \mathbf{u u})-\nabla \cdot \boldsymbol{\pi}-\frac{\mathbf{f}+\mathbf{f}_{\mathbf{s}}}{\phi^{c}}=-\frac{\rho}{\phi^{c}}\left[\mathbf{u} \frac{\partial \phi^{c}}{\partial t}+\mathbf{u u} \cdot \nabla \phi^{c}\right]
\end{gathered}
$$


Our standard, single-phase lattice-Boltzmann procedure [13,14] is capable of solving Eq. 3 and 4 if $\phi^{c}=1$ everywhere which implies that the right-hand sides of Eq. 3 and 4 are equal to zero (and also $\mathbf{f}_{\mathrm{s}}=\mathbf{0}$ in Eq. 4). For solving multiphase flow problems, the single-phase procedure has been extended with source terms: A mass source term equal to the right-hand side of Eq. 3, and a momentum source term equal to the right-hand side of Eq. 4 [15]. Evaluation of these source terms requires knowledge of the continuous phase volume fraction field $\phi^{c}$, its gradient $\nabla \phi^{c}$, and its time derivative $\partial \phi^{c} / \partial t$.

The dynamics of the spherical solid particles is governed by Newton's equations of motion

$$
\begin{gathered}
\rho_{s} \frac{\pi}{6} d^{3} \frac{d \mathbf{u}_{\mathbf{p}}}{d t}=\mathbf{F}_{\mathbf{h}}+\mathbf{F}_{\mathbf{c}}-\frac{\pi}{6} d^{3}\left(\rho_{s}-\rho_{m}\right) g \mathbf{e}_{\mathbf{x}} \\
\rho_{s} \frac{\pi}{60} d^{5} \frac{d \mathbf{\omega}_{\mathbf{p}}}{d t}=\mathbf{T}_{\mathbf{h}}+\mathbf{T}_{\mathbf{c}} \\
\frac{d \mathbf{x}_{\mathbf{p}}}{d t}=\mathbf{u}_{\mathbf{p}}
\end{gathered}
$$

with $\mathbf{u}_{\mathbf{p}}, \boldsymbol{\omega}_{\mathbf{p}}, \mathbf{x}_{\mathbf{p}}$ the linear velocity, angular velocity, and centre location of a spherical particle respectively (note that - because we are dealing with spheres - there is no need to track the angular "location" of the particles); $\mathbf{F}_{h}$ and $\mathbf{T}_{h}$ the hydrodynamic force and torque on a particle, and $\mathbf{F}_{\mathbf{c}}$ and $\mathbf{T}_{\mathbf{c}}$ the contact force and torque due to particle-particle collisions and lubrication effects.

\subsection{Modelling assumptions and procedures}

Implementation of Eqs, 3-7 in a numerical framework requires a great many assumptions and modelling steps, the sensitivity of which generally needs to be investigated. The focus of the current research is on the following three aspects: (1) Determination of the volume fractions $\phi$ and $\phi^{c}$ and their temporal and spatial derivatives on the Eulerian grid based on the size and locations $\mathbf{x}_{\mathbf{p}}$ of individual particles; (2) relating the (Lagrangian) hydrodynamic forces $\mathbf{F}_{\mathbf{h}}$ (Eq. 5) acting on a particle to the (Eulerian) body force $\mathbf{f}_{\mathrm{s}}$ (Eq. 4); (3) the size of the particles relative to the grid, i.e. the ratio $d / \Delta$. The first two aspects require 
choices for the way in which particle properties are distributed over the Eulerian grid, specifically the spatial extent of such a distribution. It is anticipated that this extent is decisive for the level of velocity fluctuations (of liquid and solids) in the sedimenting suspension. This can be appreciated given that in the hypothetical case of hydrodynamic forces being distributed uniformly over the entire periodic domain, and the solids volume fraction being considered uniform over the entire domain, the velocity fluctuation levels of liquid and solid would become zero. The more focused (i.e. the narrower) one distributes Lagrangian properties over the Eulerian grid (and vice versa), the more local flow structures appear and the higher fluctuation levels one expects. The width of the distribution process is controlled by mapping functions $[16,17]$ which are discussed in more detail below.

A major simplification in this work is that the only hydrodynamic force we will be considering is the drag force $\left(\mathbf{F}_{\mathbf{h}}=\mathbf{F}_{\mathbf{D}}\right)$. For gas-solid systems it is well established that drag is the dominating hydrodynamic force; for liquid-solid systems - with density ratios of order one - this does not necessarily need to be the case. An additional simplification is that drag is assumed to only depend on the solids volume fraction, and on the Reynolds number based on the slip velocity $\operatorname{Re}=(1-\phi)\left|\mathbf{u}-\mathbf{u}_{\mathbf{p}}\right| d / v$. We thus do not include terms in the drag expression that depend on the granular temperature (as e.g. in [18]).

In the literature there is extensive activity and debate about the manner in which the drag force depends on $\phi$ and Re. Much of the recent work is motivated by applications of gas-solid flow (e.g. gasfluidization, pneumatic conveying) where Stokes numbers are high due to the high solid over fluid density ratios. In such cases, the fluid flow time scales are much shorter than the time scales over which particle configurations change so that one can view drag as the result of the gas flowing through static (and random) assemblies of particles. Nowadays fully resolved simulations of fluid flow through assemblies of static particles are standard routine [19-22] and a great many correlations have been proposed based on such simulations. As shown by [23], the situation for liquid-solid systems that have low to intermediate Stokes numbers is very different. For liquid-solid systems the prevailing approach is the one based on the seminal experiments of solid particles settling in liquids by Richardson and Zaki 
[24]. Refinements and amendments are e.g. due to [25,26]. In this school of thought, hindered settling is described as $(1-\phi)\left|\mathbf{u}-\mathbf{u}_{\mathbf{p}}\right| / u_{\infty}=(1-\phi)^{N}$ with a well-established value for the exponent $N$ at low Reynolds numbers: $N=4.65$ [26], and relatively weak dependencies of $N$ on $\operatorname{Re}$ [26] or $\operatorname{Re}_{\infty}$ [9] for moderate Reynolds numbers. The fully resolved benchmark simulations [9], that we will be comparing our unresolved simulations with, largely confirm the validity of an exponential hindered settling scaling.

The drag force relation that is the consequence of a Richardson-Zaki type approach can be written as

$$
\mathbf{F}_{\mathbf{D}}=3 \pi \rho v d\left(\mathbf{u}-\mathbf{u}_{\mathbf{p}}\right)\left(1+0.15 \operatorname{Re}^{0.687}\right)(1-\phi)^{-\beta}
$$

with $\beta=N-2$ and a Schiller-Naumann type [11] account for finite Reynolds number drag. For (again) simplicity, and since this paper is focusing on the consequences of modelling choices on velocity fluctuations, less on average velocities, the value of the exponent has been set to the fixed value of $\beta=2.65$, i.e. independent of Re.

Mapping - as alluded to above - enters Eq. 8 via determination of $\mathbf{u}$ (which is a measure for the liquid velocity around the particle location) and $\phi$ (which is a measure for the solids volume fraction in the vicinity of the particle). The velocity $\mathbf{u}$ enters Eq. 8 directly and also via Re. Starting point for our mapping process is a "clipped fourth-order polynomial" [17] $\mu(\xi)$ which shows some resemblance to a Gaussian distribution but is computationally more efficient than a Gaussian. In one dimension:

$$
\begin{aligned}
& \mu(\xi)=\frac{15}{16}\left[\frac{\xi^{4}}{\lambda^{5}}-2 \frac{\xi^{2}}{\lambda^{3}}+\frac{1}{\lambda}\right] \text { for }-\lambda \leq \xi \leq \lambda \\
& \mu(\xi)=0 \text { for }|\xi|>\lambda
\end{aligned}
$$

The local average at location $\kappa$ over an averaging length scale $2 \lambda$ of a one-dimensional function $\alpha(\xi)$ is then determined as $\langle\alpha(\kappa)\rangle_{\lambda}=\int_{-\lambda}^{\lambda} \mu(\xi-\kappa) \alpha(\xi) d \xi$. In our simulations, $\alpha(\xi)$ is defined on an 
equidistant grid $\xi_{i}$ with spacing $\Delta$ by values $\alpha_{i}$, and we approximate $\alpha(\xi)$ in the integrant as a stair step function, i.e. $\alpha(\xi)=\alpha_{i}$ for $\xi_{i}-\frac{1}{2} \Delta \leq \xi<\xi_{i}+\frac{1}{2} \Delta$.

Such mappings can be readily extended to three dimensions:

$$
\langle\alpha(\boldsymbol{\kappa})\rangle_{\lambda}=\int_{-\lambda}^{\lambda} \int_{-\lambda}^{\lambda} \int_{-\lambda}^{\lambda} \mu\left(\xi_{1}-\kappa_{1}\right) \mu\left(\xi_{2}-\kappa_{2}\right) \mu\left(\xi_{3}-\kappa_{3}\right) \alpha(\xi) d \xi_{1} d \xi_{2} d \xi_{3}
$$

Equation 10 is used to determine the liquid velocity $\mathbf{u}$ and solids volume fraction $\phi$ from grid values in the expression for the drag force (Eq. 8). We will check the impact filter length $\lambda$ has on the simulation results.

An inverse mapping operation is required to couple back the hydrodynamic force $-\mathbf{F}_{\mathbf{D}}$ to the liquid. This involves a distribution of the force over grid points surrounding a particle. A similar operation applies for determining the solids volume fraction on the grid: the volume of each particle will be distributed over surrounding grid cells.

Given the discrete nature of $\alpha(\xi)$, Eq. 10 can be written as $\langle\alpha(\mathbf{\kappa})\rangle_{\lambda}=\sum_{i} \sum_{j} \sum_{k} \eta_{i j k} \alpha_{i j k}$ with $i, j, k$ discrete coordinates in $x, y$, and $z$-direction respectively, and $\eta_{i j k}$ coefficients following from integrating the mapping function. The coefficients $\eta_{i j k}$ are only non-zero on grid points within a volume of $(2 \lambda)^{3}$ around $\boldsymbol{\kappa}$, and $\sum_{i} \sum_{j} \sum_{k} \eta_{i j k}=1$ since for $\alpha$ uniform $\langle\alpha\rangle_{\lambda}=\alpha$.

The same coefficients $\eta_{i j k}$ are used to distribute particle properties to the grid. The drag force on one of the particles $\left(\mathbf{F}_{\mathbf{D}}\right)$ contributes to the body force on the fluid $\mathbf{f}_{\mathrm{s}}$ (see Eq. 4) in grid cell $i, j, k$ by an amount $-\frac{1}{\Delta^{3}} \mathbf{F}_{\mathbf{D}} \eta_{i j k}$; that same particle contributes to the solids volume fraction in cell $i, j, k$ by an amount $\frac{\pi d^{3}}{6 \Delta^{3}} \eta_{i j k}$. For the total interaction force and the total solids volume fraction in each grid cell $(i, j, k)$ contributions from all particles need to be added up. 
Applying the lattice-Boltzmann method for incompressible flow requires that liquid velocities should stay well below the speed of sound [27]. This implies that - measured in lattice units (lattice distance per time step; $\Delta / \Delta t$ ) - liquid speeds need to be limited to order 0.1 (liquid travels typically less than $0.1 \Delta$ per $\Delta t$ ). As a result, particle speeds are limited to order 0.1 as well. Together with the distribution through mapping functions of solids volume over clusters of lattice cells around particles this leads to a smoothly varying (in three-dimensional space and time) solids volume fraction field $\phi(x, y, z ; t)$ and thus liquid volume fraction field $\phi^{c}(x, y, z ; t)$ which are amenable to numerical differentiation to determine $\nabla \phi^{c}$, and $\partial \phi^{c} / \partial t$, as encountered in Eqs. 3 and 4. We use central differences for $\nabla \phi^{c}$, and an Euler backward method for $\partial \phi^{c} / \partial t$.

\subsection{Particle dynamics modelling}

Now we turn to particle dynamics, i.e. the way Eqs. 5-7 are dealt with. In Section 2.3 it was already explained how $\mathbf{F}_{\mathbf{h}}$ (equal to $\mathbf{F}_{\mathbf{D}}$ ) was determined. The contact force $\mathbf{F}_{\mathbf{c}}$ consists of two parts: soft-sphere collision forces $\mathbf{F}_{\text {ssc }}$ and lubrication forces $\mathbf{F}_{\text {lub }}$. Both forces are assumed to be radial forces, meaning that they act on the line connecting the two sphere centres involved in the contact. We will not be considering tangential contact forces and contact torques, meaning that $\mathbf{T}_{\mathbf{c}}=\mathbf{0}$ in Eq. 6.

For determining $\mathbf{F}_{\text {ssc }}$, overlap distances $\delta$ of spheres are detected. Once two spheres overlap, they are given a (equal and opposite) linear, elastic repulsive force with magnitude $\left|\mathbf{F}_{\text {ssc }}\right|=k \delta$. The spring constant $k=\pi^{2} m / t_{c}^{2} \quad\left(m=\pi \rho_{s} d^{3} / 6\right.$ is the mass of a particle $)$ is largely a numerical parameter and is chosen such that a typical collision time is 10 time steps $t_{c}=10 \Delta t$ [23]. In a typical simulation this results in maximum overlapping distance of not more than $10^{-2} \mathrm{~d}$. This approach leads to elastic (restitution coefficient $e=1$ ), smooth (i.e. frictionless) collisions between particles. 
Lubrication forces are the result of relative velocity between closely spaced particles. When two particles approach, a liquid film between them needs to be squeezed out which brings about a repulsive force between the particles, for similar reasons, an attractive force arises when two particles separate. Radial lubrication forces are included in the simulations. The low-Reynolds number, analytical expression for the magnitude of the radial lubrication force between two equally sized spheres is $\left|\mathbf{F}_{\text {lub }}\right|=-\frac{3}{8} \pi v \rho d^{2} \Delta u_{p, r} / s$ with $s$ the distance between the two particle surfaces, and $\Delta u_{p, r}$ the relative velocity of the two particles in radial direction (with $\Delta u_{p, r}>0$ separation, hence the minus sign in the expression for the lubrication force) [28]. In the simulations we have limited the distance over which the lubrication force acts to $s<d / 10$ and changed the expression to $\left|\mathbf{F}_{\text {lub }}\right|=-\frac{3}{8} \pi v \rho d^{2} \Delta u_{p, r}\left(\frac{1}{s}-\frac{10}{d}\right)$ [10]. To avoid divergence when particle touch $(s=0)$ the lubrication is saturated, i.e. does not increase anymore upon approach, if $s<0.01 d[10]$.

The hydrodynamic torque ( $\mathbf{T}_{\mathbf{h}}$ in Eq. 6) on a particle is determined from the vorticity of the liquid in direct vicinity of the particle $(\boldsymbol{\omega})$ and the rotation rate of the particle $\left(\boldsymbol{\omega}_{\mathbf{p}}\right): \mathbf{T}_{\mathbf{h}}=\pi \rho v d^{3}\left(\frac{1}{2} \boldsymbol{\omega}-\boldsymbol{\omega}_{\mathbf{p}}\right)$. This is a creeping flow approximation. The rotation of the particle is not coupled back to the liquid. Since the rotation does also not play a role in particle-particle collisions $\left(\mathbf{T}_{\mathbf{c}}=\mathbf{0}\right)$, particle rotation has no effect on the overall dynamics of the suspension. We do solve Eq. 6 as a matter of completeness.

The equations of motion (Eq. 5-7) are solved by means of a split derivative time integration which has been discussed in detail in [29].

\section{Results}

\subsection{Base-case}


First a base-case will be introduced. This allows us to explain how the simulations results were analysed and will give us the opportunity to give visual impressions of the flow system. The dimensionless parameters of the base case are $\phi=0.350, \rho_{s} / \rho=4.0, \operatorname{Re}_{\infty}=1.85$, and $d / n x=1 / 24$.

The particle-resolved simulation of this case had a spatial resolution such that $d=12 \Delta$. Results of this case, that also have been presented elsewhere [9], are compared to unresolved simulations with particle diameters of $d=2 \Delta, \Delta$, and $0.75 \Delta$ respectively. In the unresolved simulations the width of the mapping function has - initially - been fixed to $\lambda=1.5 d$ as e.g. suggested by [17]. In Figures 1 and 2 we show cross sections through the flow system. To facilitate comparison, the velocities have been shifted so that all cases shown in Figures 1 and 2 have volume-averaged liquid velocity in the $x$-direction equal to zero.

In Figure 1, resolved and unresolved simulations are compared. In the resolved simulation shown in full resolution (top panel), the interaction between liquid and solid is clearly visible. One observes liquid squeezing through the open space between the particles, and the liquid flow being deflected by the particles. Each vector we show in the middle panel of Figure 1 is the average of a cluster of $6 \times 6 \times 6$ vectors of the fully resolved flow, i.e. we have spatially filtered the fully resolved flow to a resolution that is the same as that of an unresolved simulation with $d=2 \Delta$. Results of the latter are shown in the lower panel of Figure 1. The clear interaction between liquid and solid, still visible in the filtered resolved field, is to a large extent lost in the unresolved field. This is not a surprise given the schematizations in the unresolved simulations as discussed above. Comparing middle and lower panel more gobally, one sees similar levels of variation of liquid velocities and somewhat higher absolute particle velocities (more red particles) in the lower panel. Figure 2 shows comparisons among unresolved simulations, with the particle size over grid size decreasing from top to bottom.

It is clear that much of the flow physics captured in the particle-resolved simulations is lost in the particle-unresolved simulations. In resolved simulations, hydrodynamic interactions between particles are largely captured (only for particles that are very close, lubrication modelling enters). In unresolved 
simulations, these interactions are parameterized in a drag force correlations that involves $\phi$ (Eq. 8) and also the interactions enter through $\phi^{c}=1-\phi$ that is contained in the liquid mass and momentum balances (Eqs. 3 and 4). Note that in the current approach only the drag force is included, other type forces will certainly contribute further to interactions. Also note that as a result of mapping, $\phi$ is a spatial average over a length scale $2 \lambda$ which usually equals a few particle diameters.

The pragmatic approach we will be following is to try and establish how sensitive the outcomes of unresolved simulations of settling particles are with respect to the two main numerical parameters we have: $d / \Delta$ and $\lambda / d$. With regards to the outcomes, we will focus on the superficial slip velocity $(1-\phi)\left|\mathbf{u}-\mathbf{u}_{\mathbf{p}}\right|$ and on the velocity fluctuations in the $x$ (direction of gravity) and $y$ and $z$ directions of particles as well as liquid. For the base-case we first establish these quantities for the particle-resolved simulation. Figure 3 shows quasi-steady time series of the volume-averaged quantities $u_{s}=\left\langle(1-\phi)\left|\mathbf{u}-\mathbf{u}_{\mathbf{p}}\right|\right\rangle, u_{x}^{\prime}=\sqrt{\left\langle u_{x}^{2}\right\rangle-\left\langle u_{x}\right\rangle^{2}}, u_{y}^{\prime}=\sqrt{\left\langle u_{y}^{2}\right\rangle}, u_{z}^{\prime}=\sqrt{\left\langle u_{z}^{2}\right\rangle}, u_{p x}^{\prime}=\sqrt{\left\langle u_{p x}^{2}\right\rangle-\left\langle u_{p x}\right\rangle^{2}}, u_{p y}^{\prime}=\sqrt{\left\langle u_{p y}^{2}\right\rangle}$, $u_{p z}^{\prime}=\sqrt{\left\langle u_{p z}^{2}\right\rangle}$ with the brackets \langle\rangle indicating volume averaging. There is a clear anisotropy in the velocity fluctuations, with the $x$-velocities fluctuating stronger by a factor of approximately two (for liquid as well as for particles). The particles fluctuations being somewhat weaker than the liquid fluctuations is due to density of the particles being four times higher than the liquid density. The slip velocity is at a level of $u_{s} \approx 0.135 u_{\infty}$ which demonstrates the hindered settling effect [24].

Time series of unresolved particle simulation - counterparts of the particle-resolved time series in Figure 3 - for a mapping function width $\lambda=1.5 d$ are given in Figure 4 . The general observations are similar as for the resolved simulations: $x$-fluctuations stronger than $y$ and $z$ fluctuations; liquid velocity fluctuations stronger than particle velocity fluctuations. Spatial resolution, as expressed by the ratio $d / \Delta$ has significant impact on the results. Fluctuation levels decrease with decreasing resolution. Also the difference between liquid and solids fluctuations reduces with decreasing resolution. 
The slip velocity is remarkably stable in all simulations shown in Figure 4. It is consistently (some $10 \%$ ) higher than in the resolved simulation: $u_{s} \approx 0.15 u_{\infty}$, almost independent of the spatial resolution. As we will see shortly, the $u_{s} \approx 0.15 u_{\infty}$ also is hardly dependent on the width $\lambda$ of the mapping function (if $\lambda>d)$. In the extreme case of uniform mapping $(\lambda \rightarrow \infty)$ we find from the simulations $u_{s}=0.153 u_{\infty}$. The latter value is consistent with the use of Eq. 8 as the expression for the drag force. This consistency has been detailed in Appendix A.

The simulation data have been further reduced to time averages taken over quasi-steady portions of the simulations. Still for the base-case, these data are presented in Figure 5. The slip velocity shows only a weak dependence on the numerical parameters $d / \Delta$ and $\lambda / d$ if $\lambda / d>1$. The reduction of slip velocity at $\lambda / d=1$ at the lower resolutions is the result of the now very focused reaction force acting from particles to lquid which locally strongly drags down the liquid.

Qualitatively, the general observations of velocity fluctuations in the resolved simulations are recovered by the unresolved simulations: Fluctuations in gravity direction are a factor of approximately two larger than in the lateral directions, and liquid fluctuations are stronger compared to particle fluctuations. Also the order of magnitude of the fluctuations in the unresolved simulations corresponds to those in the resolved simulations.

There is a clear trend with the numerical parameters though. The strongest is the one with $\lambda / d$. Fluctuations decrease with increasing $\lambda / d$. This is to be expected, the wider the influence of one particle is felt, the more it will behave in coherence with its neighbouring particles (and - as we argued above - if $\lambda / d$ goes to infinity the fluctuations will disappear). According to Figure 5, the best agreement between fluctuations in unresolved and resolved simulations is reached for $\lambda / d$ in the range 1.0 to 1.5 . It is interesting to note that Capecelatro and Desjardins [30] recommend a resolution at the level of $\lambda / d=3$. 
Given the sensitivity of the slip velocity at $\lambda / d=1$ for the resolution $(d / \Delta)$, it was decided to continue working with $\lambda / d=1.5$ and to study the performance of unresolved simulations with this setting for a range of solids volume fractions and $\mathrm{Re}_{\infty}$ values.

\subsection{Slip velocity and particle fluctuation levels}

In Figure 6 the results of the parameter study have been summarized. Since the trends in particle and liquid velocity fluctuations was the same, only particle fluctuations levels are reported. The downward trend of all variables on display with an increasing solids volume fraction is no surprise and can be summarized as more hindrance between particles for denser suspensions. The agreement between resolved and unresolved simulations we consider fair for the lower part of the Reynolds number range studied. For $\operatorname{Re}_{\infty} \geq 10$, however, we see that the unresolved simulations strongly deviate from the resolved ones. Specifically at low $\phi$ the fluctuations come out too high in the unresolved simulations.

\subsection{A sample mixing tank result}

In order to give a sense of the potential of the above described numerical approach to agitated solid-liquid systems, we here give a brief account of simulations of a particle suspension process in a mixing tank. The tank, as displayed in Figure 7, has the same geometry as the one we previously used for particleresolved simulations [31]. The "pitched-blade" impeller rotates such that it pumps liquid in the downward direction. The liquid flow in the tank is characterized by a Reynolds number that is defined as $\operatorname{Re}_{m x} \equiv n D^{2} / v$ (with $D$ the impeller diameter, and $n$ its angular velocity in revolutions per unit time) and has a value of 1440 . Without solid particles present this would be a mildly turbulent flow. In this tank we place $N=290,000$ solid, spherical particle with a diameter $d=0.0229 D$. The overall solids volume fraction of this system is $\phi=N \pi d^{3} /\left(6 W^{2} H\right)=0.138$. The density ratio is $\rho_{s} / \rho=2.5$. A useful dimensionless parameter that to a significant extent characterizes the suspensions process is a modified 
Shields number [30] $\theta=\rho n^{2} D^{2} /\left[g d\left(\rho_{s}-\rho\right)\right]$. It is the ratio of inertial stress (that scales with $\rho n^{2} D^{2}$ and is the mechanism responsible for suspending the particles) and net gravity. In the simulation presented here $\theta=48$.

The sequence of instantaneous realizations in Figure 8 reveals some interesting features of this solid-liquid flow. In the first place it shows - in the initial stages - the way the stream of liquid coming off the impeller in the downward direction is eroding the bed of particles. In the later stages a cone of particles forms under the impeller. This cone the result of the particles influencing the overall circulation structure in the tank (a solid-to-liquid coupled phenomenon): the liquid stream generated by the impeller is deflected in outward radial direction by the presence of a dense packing of particles under the impeller. This stream is therefore not able to truly mobilize and suspend the particles in the cone. The stream does continuously erode the surface of the cone.

Experiments are very much needed to assess the level of realism of these types of simulations. Matching the refractive index of liquid and (glass) particles would allow for performing experiments that look through the suspension with optical methods.

\section{Conclusions}

In this paper we have described and subsequently assessed an Eulerian-Lagrangian simulation method for solid-liquid suspensions. A typical feature is that the method allows for the particle size to be of the same order of magnitude as the Eulerian grid spacing. Additionally, the assessment was focused on the - from a practical point of view - very relevant range of high solids volume fractions $(\phi>0.30)$ and on velocity fluctuations.

By comparing with fully resolved simulations, it was concluded that a reasonable choice for the width of the mapping function that facilitates two-way communication between the particles and the grid is one and a half particle diameter $(\lambda / d=1.5)$. 
Results between resolved and unresolved simulations show fair agreement in terms of superficial slip velocity between solid and liquid and in terms of particle velocity fluctuation levels except for $\operatorname{Re}_{\infty} \geq 10$. At that stage, the unresolved simulations tend to over-predict slip velocities as well as velocity fluctuations. One route to investigate this further is by considering more sophisticated drag relations that e.g. include a Reynolds number based on granular temperature [18] or the Stokes number [23]. If this would improve slip velocity predictions, it might at the same time improve fluctuation levels. The reason for this is that the over-prediction of fluctuations and slip velocity happen in the same part of the $\left(\phi, \mathrm{Re}_{\infty}\right)$ parameter space. Particles moving with less slip through the liquid will reduce local forces on the liquid and thereby might reduce liquid and particle fluctuations.

\section{Appendix A}

Here we discuss hindered settling velocity ratios in case mapping is uniform, i.e. $\lambda \rightarrow \infty$. The force balance over a single particle in the $x$-direction is

$$
g\left(\rho_{s}-\rho\right) \frac{\pi}{6} d^{3}=3 \pi \rho v d u_{\infty}\left(1+0.15 \mathrm{Re}_{\infty}^{0.687}\right)
$$

The force balance over one particle in a swarm of particles in $x$-direction is

$$
g\left(\rho_{s}-\rho_{m}\right) \frac{\pi}{6} d^{3}=3 \pi \rho v d\left|\mathbf{u}-\mathbf{u}_{\mathbf{p}}\right|\left(1+0.15 \operatorname{Re}^{0.687}\right)(1-\phi)^{-\beta}
$$

With $\quad \rho_{s}-\rho_{m}=(1-\phi)\left(\rho_{s}-\rho\right) \quad$ and $\quad u_{s}=(1-\phi)\left|\mathbf{u}-\mathbf{u}_{\mathbf{p}}\right| \quad$ Eq. A2 can be written as $g\left(\rho_{s}-\rho\right) \frac{\pi}{6} d^{3}=3 \pi \rho v d u_{s}\left(1+0.15 \operatorname{Re}^{0.687}\right)(1-\phi)^{-\beta-2}$. Dividing this by Eq. A1 leads to

$$
\frac{u_{s}}{u_{\infty}}=\frac{\operatorname{Re}}{\operatorname{Re}_{\infty}}=(1-\phi)^{\beta+2} \frac{\left(1+0.15 \mathrm{Re}_{\infty}^{0.687}\right)}{\left(1+0.15 \mathrm{Re}^{0.687}\right)}
$$

In the base case $\phi=0.35$ and $\operatorname{Re}_{\infty}=1.85$. Solving this non-linear equation in $\operatorname{Re}($ with $\beta=2.65$ ) gives $\frac{u_{s}}{u_{\infty}}=\frac{\operatorname{Re}}{\operatorname{Re}_{\infty}}=0.153$ which also was the result of a (unresolved) simulation with $\lambda \rightarrow \infty$. 


\section{References}

[1] Z.K. Nagy, G. Fevotte, H. Kramer, L.L. Simon, Recent advances in the monitoring, modelling and control of crystallization systems, Chem. Eng. Res. Des. 91 (2013) 1903-1922.

[2] J.M. Buffington, D.R. Montgomery, A systematic analysis of eight decades of incipient motion studies, with special reference to gravel-bedded rivers, Water Resources Res. 33 (1997) 19932029.

[3] M.P. Dudukovic, F. Larachi, P.L. Mills, Multiphase reactors - revisited, Chem. Eng. Sc. 54 (1999) 1975 - 1995.

[4] R.G. Gillies, C.A. Shook, Modeling high concentration settling slurry flows, Can. J. Chem. Eng. 78 (2000) 709-716.

[5] S. Laín, M. Sommerfeld, Characterisation of pneumatic conveying systems using the Euler/Lagrange approach, Powder Technology 235 (2013) 764 - 782.

[6] A.G. Kidanemariam, M. Uhlmann, Direct numerical simulation of pattern formation in subaqueous sediment, J. Fluid Mech. 750 (2014) R2 1 - 13.

[7] N.G. Deen, S.H.L. Kriebitzsch, M.A. van der Hoef, J.A.M. Kuipers, Multi-scale modeling of dispersed gas-liquid two-phase flows, Chem. Eng. Sc. 81 (2012) 329 - 344.

[8] J.J. Derksen, Numerical simulation of solids suspension in a stirred tank, AIChE J. 49 (2003) 2700 2714.

[9] J.J. Derksen, Simulations of scalar dispersion in fluidized solid-liquid suspensions, AIChE J. 60 (2014) 1880 - 1890.

[10] J.J. Derksen, S. Sundaresan, Direct numerical simulations of dense suspensions: wave instabilities in liquid-fluidized beds, J. Fluid Mech. 587 (2007) 303 - 336.

[11]L. Schiller, A. Naumann, Uber die grundlagenden Berechnungen bei der Schwerkraftaufbereitung, Ver. Deut. Ing. Z. 77 (1933) 318-320.

[12] J.J. Derksen, Simulations of hindered settling of flocculating spherical particles, Int. J. Multiphase Flow 58 (2014) 127 - 138.

[13] J.A. Somers, Direct simulation of fluid flow with cellular automata and the lattice-Boltzmann equation, App. Sci. Res. 51 (1993) 127-133.

[14] J.G.M. Eggels, J.A. Somers, Numerical simulation of free convective flow using the latticeBoltzmann scheme, Int J Heat Fluid Flow 16 (1995) 357-64.

[15] R. Sungkorn, J.J. Derksen, Simulations of dilute sedimenting suspensions at finite-particle Reynolds numbers, Phys. Fluids 24 (2012) 123303. 
[16] A. Kitagawa, Y. Murai, F. Yamamoto, Two-way coupling of Eulerian-Lagrangian model for dispersed multiphase flows using filtering functions, Int. J. Multiphase Flow 27 (2001) 21292153.

[17] N.G. Deen, M. van SintAnnaland, J.A.M. Kuipers, Multi-scale modeling of dispersed gas-liquid twophase flow, Chem. Eng. Sc. 59 (2004) 1853 - 1861.

[18] J.J. Wylie, D.L. Koch, A,J,C. Ladd, Rheology of suspensions with high particle inertia and moderate fluid inertia, J. Fluid Mech. 480 (2003) 95-118.

[19] R.J. Hill, D.L. Koch, A.J.C. Ladd, Moderate-Reynolds-number flows in ordered and random arrays of spheres, J. Fluid Mech. 448 (2001) 243-278.

[20] D. Kandhai, J.J. Derksen, H.E.A. van den Akker, Interphase drag coefficients in gas-solid flows, AIChE J. 49 (2003) 1060-1065.

[21] M.A. van der Hoef, R. Beetstra, J.A.M. Kuipers, Lattice-Boltzmann simulations of low-Reynoldsnumber flow past mono- and bidisperse arrays of spheres: results for the permeability and drag force, J. Fluid Mech. 528 (2005) 233-254.

[22] S. Tenneti, R. Garg, S. Subramaniam, Drag law for monodisperse gas-solid systems using particleresolved direct numerical simulation of flow past fixed assemblies of spheres, Int. J. Multiphase Flow 37 (2011) 1072-1092.

[23] G.J. Rubinstein, J.J. Derksen, S. Sundaresan, Lattice-Boltzmann simulations of low-Reynolds number flow past fluidized spheres: effect of Stokes number on drag force, J. Fluid Mech. 788 (2016) $576-601$.

[24] J.F. Richardson, W.N. Zaki, 1954 Sedimentation and fluidisation. Part 1. Trans. Inst. Chem. Engrs. 32 (1954) 35-53.

[25] C.Y. Wen, Y.H. Yu, Mechanics of fluidization. Chem. Engng. Prog. 62 (1966) 100-111.

[26] R. Di Felici, The voidage function for fluid-particle interaction systems, Int. J. Multiphase Flow 20 (1994) $155-159$.

[27] S. Succi, The lattice Boltzmann equation for fluid dynamics and beyond, Clarendon Press, Oxford (2001).

[28] S. Kim, S.J. Karrila, Microhydrodynamics: Principles and Selected Applications, ButterworthHeinemann (1991).

[29] O. Shardt, J.J. Derksen, Direct simulations of dense suspensions of non-spherical particles, Int. J. Multiphase Flow 47 (2012) 25 - 36.

[30] J. Capecelatro, O. Desjardins, An Euler-Lagrange strategy for simulating particle-laden flows, - J. Comp. Phys. 238 (2013) $1-31$. 
[31] J.J. Derksen, Highly resolved simulations of solids suspension in a small mixing tank, AIChE J. 58 (2012) $3266-3278$. 


\section{Figures}

Figure 1. Base-case. Instantaneous cross sections through the simulation domain. Liquid velocity vectors, particle locations, and particle speeds. Gravity points to the left. The average liquid velocity is zero. Top panel: resolved simulation with $d=12 \Delta$ showing the full resolution of the liquid flow; middle panel: the same realization with now the liquid flow filtered to the same resolution as the bottom panel; bottom panel: unresolved simulation with $d=2 \Delta$, and $\lambda=1.5 d$. The reference vector (see bottom, left) applies to all three panels.

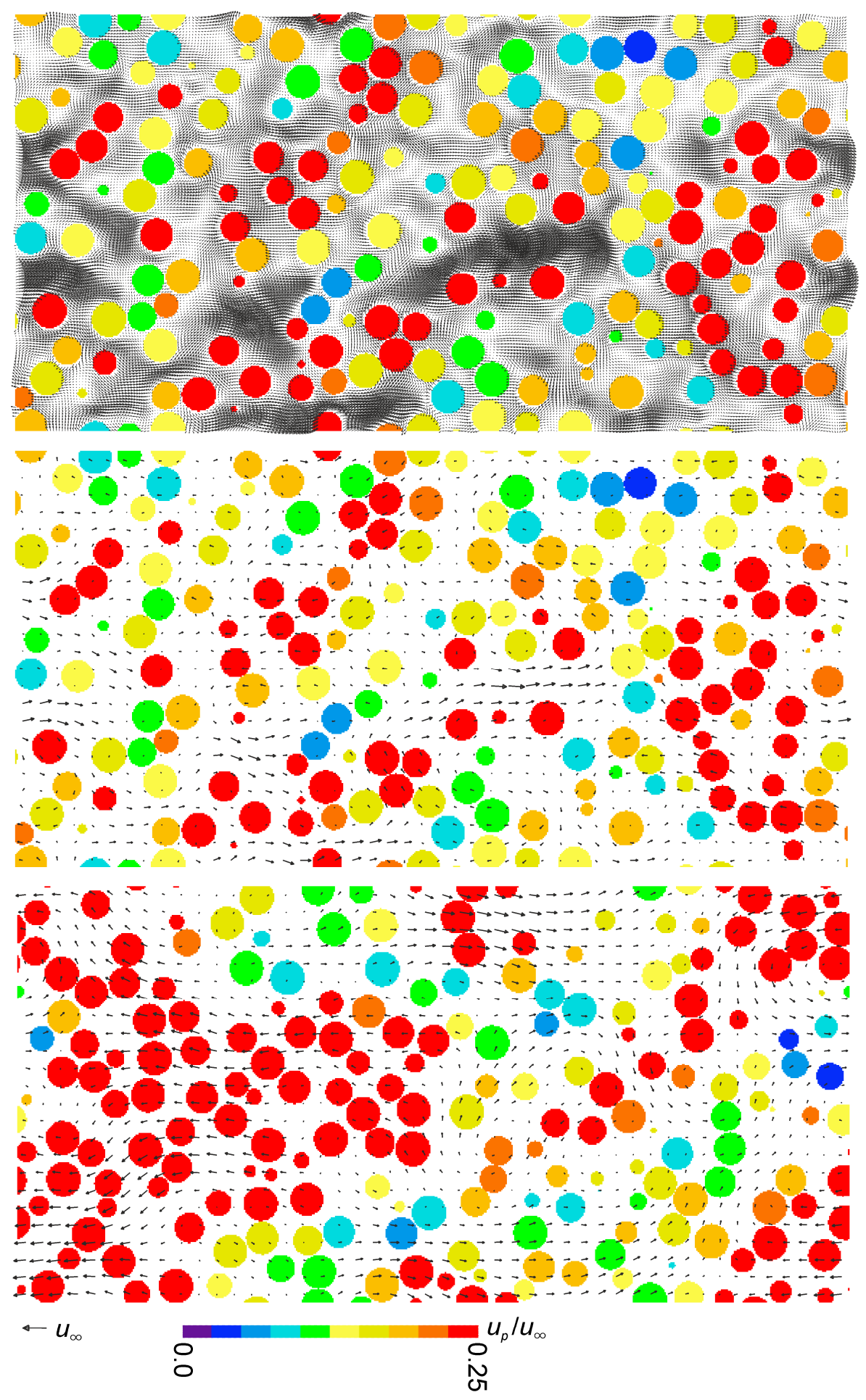


Figure 2. Base-case. Instantaneous cross sections through the simulation domain. Liquid velocity vectors, particle locations, and particle speeds. Gravity points to the left. Unresolved simulations. Top: $d=2 \Delta$ (same figure as Fig. 1, bottom); middle $d=\Delta$; bottom: $d=0.75 \Delta$. All three cases have $\lambda=1.5 d$.

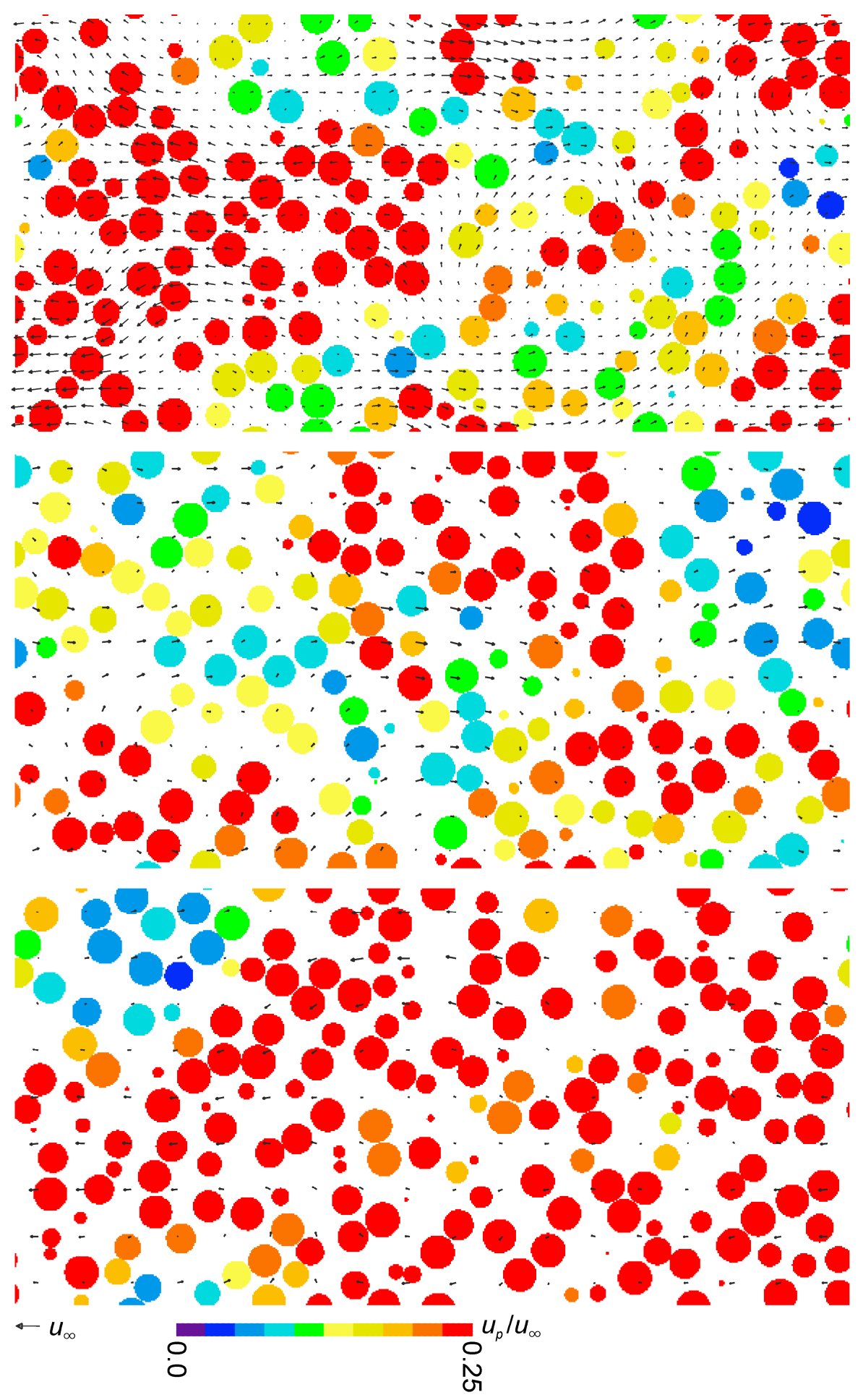


Figure 3. Base-case; particle-resolved simulations. Time series of the volume-averaged velocity fluctuation levels as well as of the slip velocity $u_{s}$. Left: particles; right: liquid.

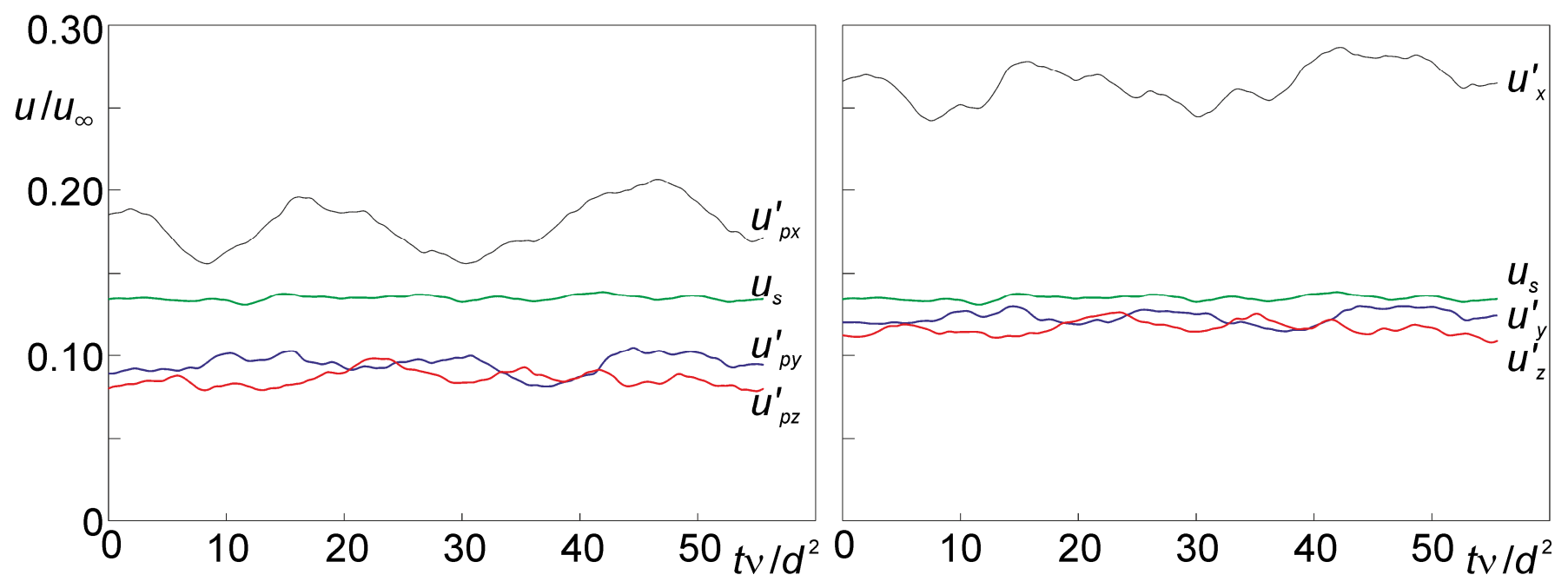


Figure 4. Base-case; unresolved simulations. Time series of the volume-averaged velocity fluctuation levels as well as of the slip velocity $u_{s}$. Left: particles; right: liquid. All results obtained for $\lambda=1.5 d$. Decreasing resolution from top to bottom: $d=2 \Delta, \Delta, 0.75 \Delta$ respectively.
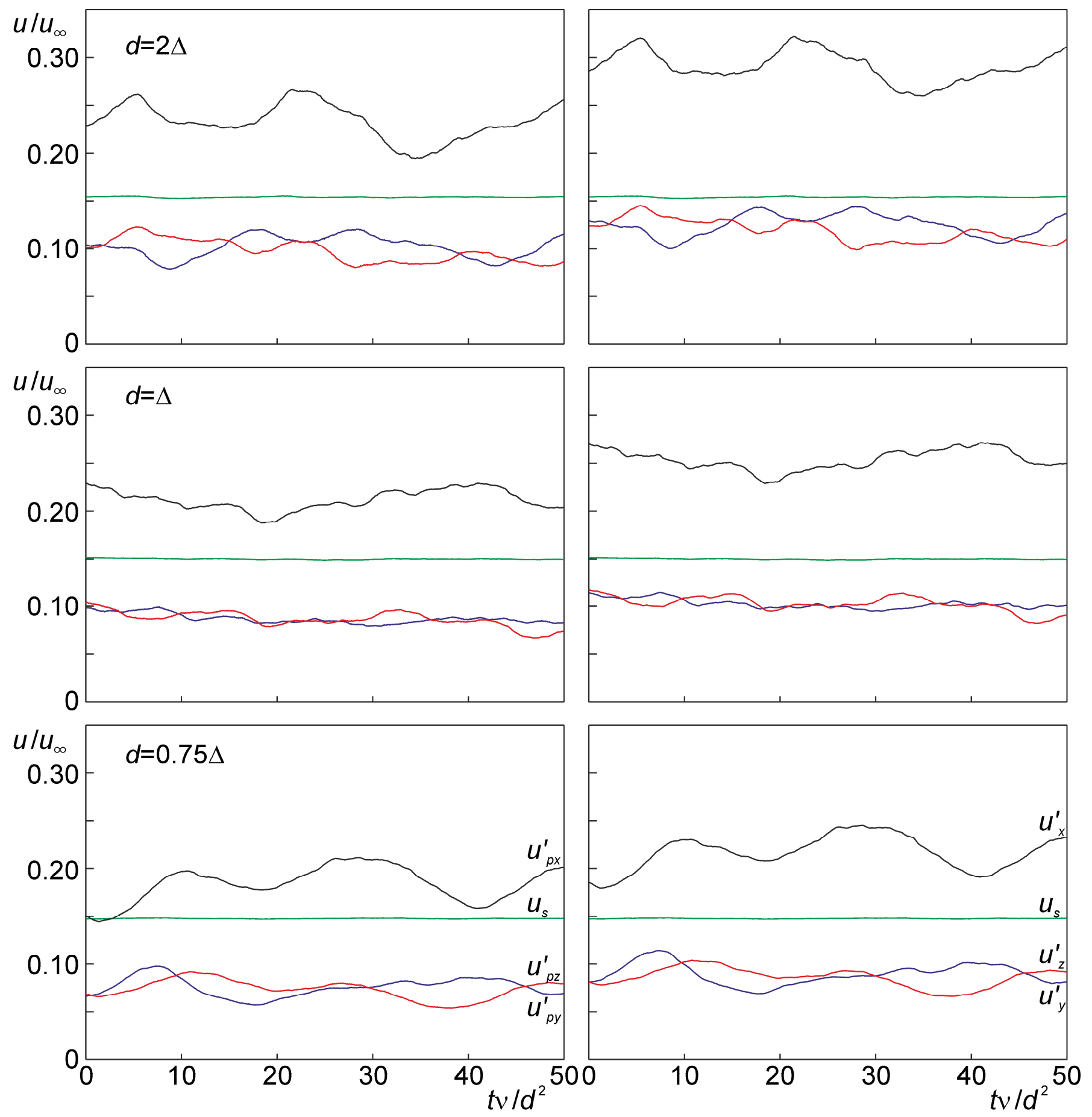
Figure 5. Base-case; unresolved simulations. Time-averaged values for (from left to right) slip velocity, liquid velocity fluctuations, and particle velocity fluctuations as a function of mapping function width $\lambda$. The different symbols signify particle size relative to grid spacing (as indicated). Black symbols relate to $x$-components, red and blue in the center panel to $y$ and $z$ respectively, red in the right panel to $y$ and $z$ averaged. The horizontal lines are resolved base-case results.
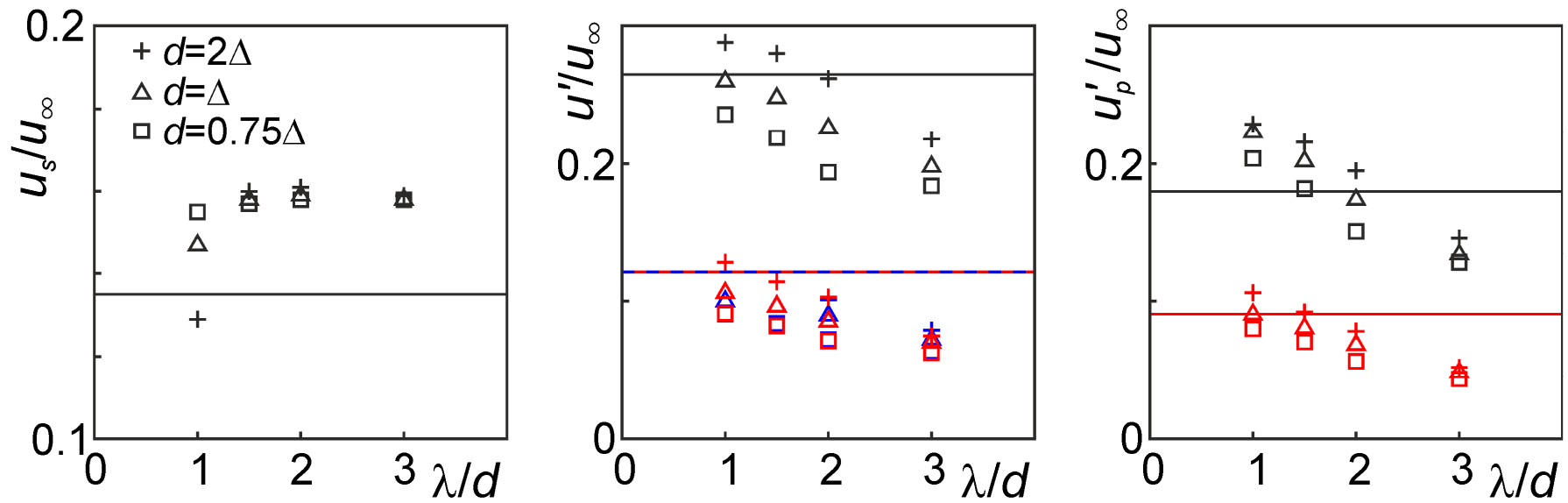
Figure 6. Particle-resolved versus particle-unresolved simulations. From left to right: $u_{s}, u_{p x}^{\prime}$, and $u_{p y z}^{\prime}=\frac{1}{2}\left(u_{p y}^{\prime}+u_{p z}^{\prime}\right)$ respectively. Increasing $\operatorname{Re}_{\infty}$ from top to bottom as indicated. The indicate resolved simulations; $\Delta$ is unresolved. In all simulations $d=\Delta$ and $\lambda=1.5 d$.
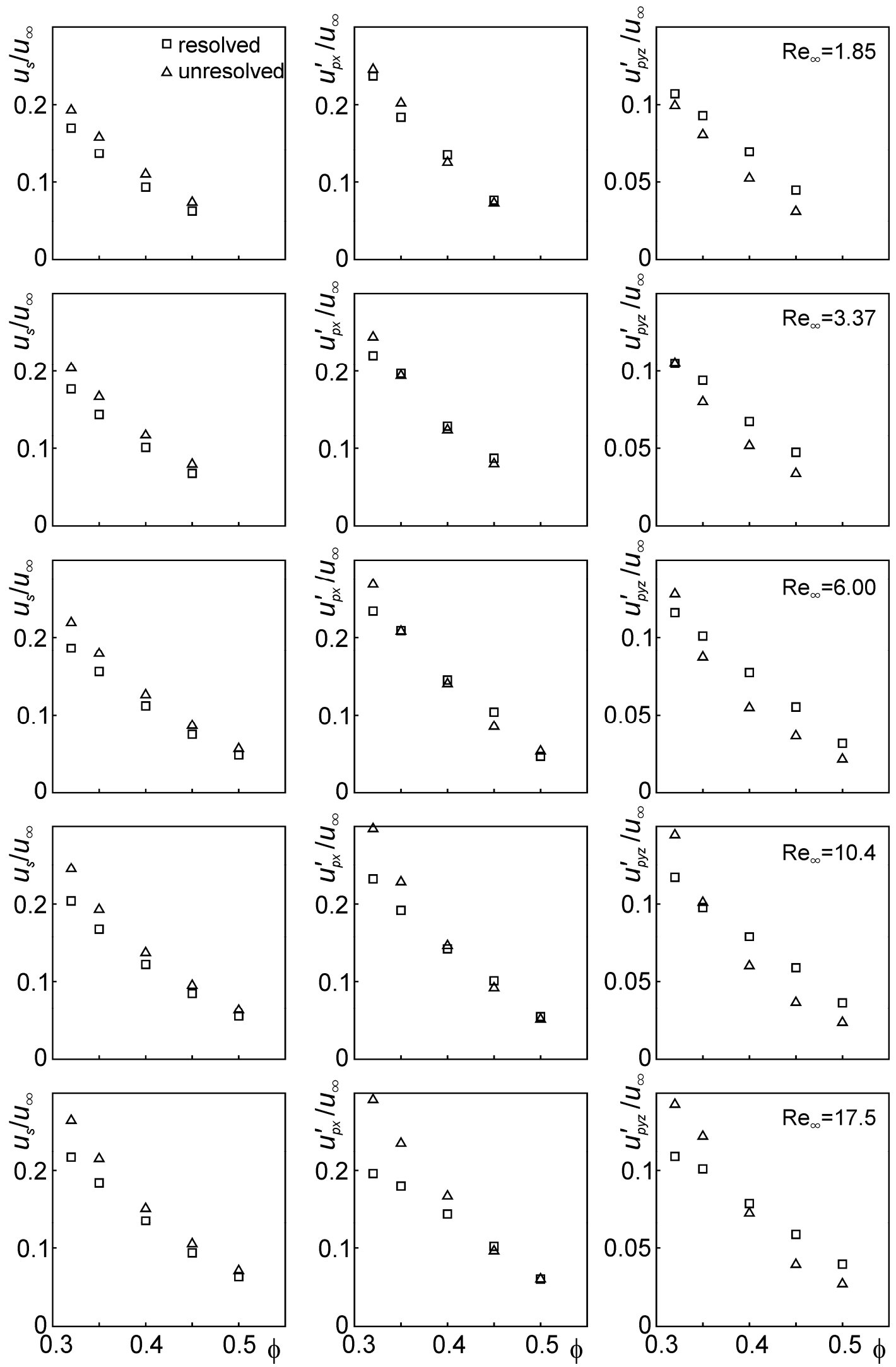
Figure 7. Initial situation for solid suspension simulation. Spherical particles (all with diameter $d$ ) fill up the lower portion of a square tank with side length $W$ and height $H=0.83 W$. The impeller has a diameter $D=0.4 W=43.6 d$. The number of particles is such that the overall, tank-averaged solids volume fraction is $\phi=0.138$.

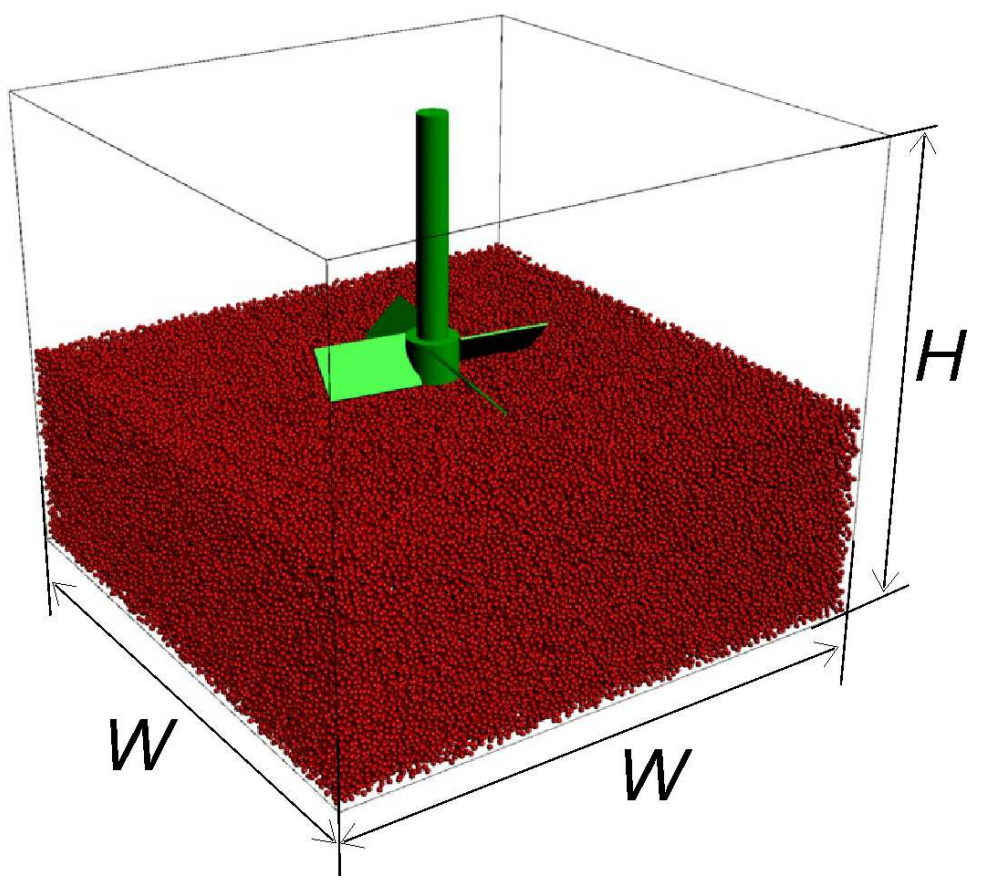


Figure 8. Start of the solids suspension process in the mixing tank. Only the particles with their center in a vertical slice with thickness $10 \mathrm{~d}$ are displayed. The colors in the plane perpendicular to the slice indicate liquid speed relative to the impeller tip speed $\mathrm{v}_{\text {tip }}$. The snapshots are 1, 4, 16 and 40 impeller revolutions after starting the impeller (from panel (a) to (d) respectively).
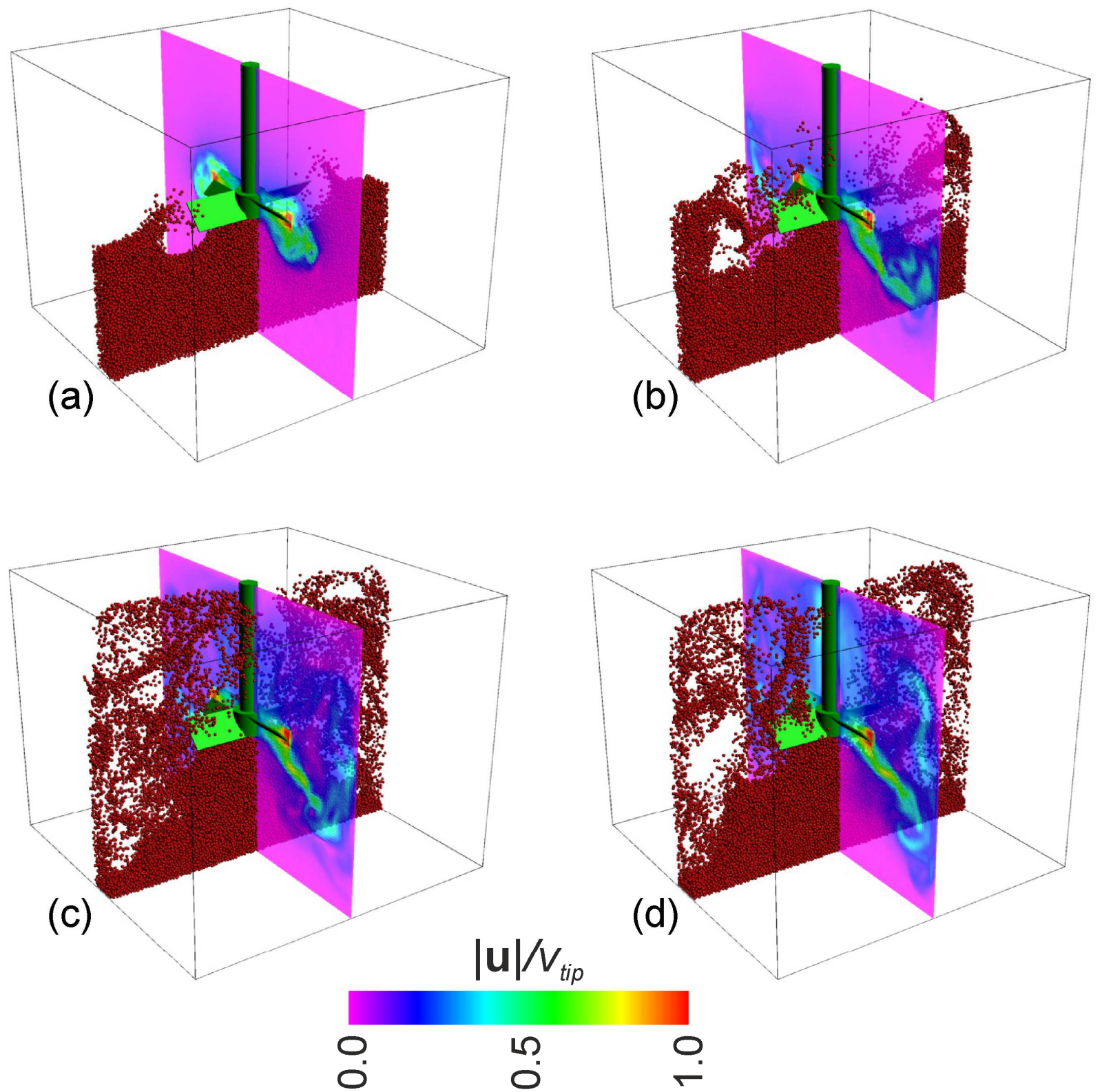